\title{
Electrical neuroimaging based on biophysical constraints
}

\author{
Rolando Grave de Peralta Menendez, ${ }^{\text {a,* }}$ Micah M. Murray, ${ }^{\text {a,b,c }}$ Christoph M. Michel, ${ }^{a}$ \\ Roberto Martuzzi, ${ }^{\mathrm{c}}$ and Sara L. Gonzalez Andino ${ }^{\mathrm{a}}$ \\ ${ }^{a}$ Functional Brain Mapping Laboratory, Neurology Department, University Hospital of Geneva, 1211 Geneva, Switzerland \\ ${ }^{\mathrm{b}}$ Rehabilitation Clinic, Department of Clinical Neurosciences, University Hospital of Geneva, 1211 Geneva, Switzerland \\ ${ }^{\mathrm{c}}$ Department of Diagnostic and Interventional Radiology, CHUV, 1011 Lausanne, Switzerland
}

Received 6 May 2003; revised 25 September 2003; accepted 26 September 2003

\begin{abstract}
This paper proposes and implements biophysical constraints to select a unique solution to the bioelectromagnetic inverse problem. It first shows that the brain's electric fields and potentials are predominantly due to ohmic currents. This serves to reformulate the inverse problem in terms of a restricted source model permitting noninvasive estimations of Local Field Potentials (LFPs) in depth from scalprecorded data. Uniqueness in the solution is achieved by a physically derived regularization strategy that imposes a spatial structure on the solution based upon the physical laws that describe electromagnetic fields in biological media. The regularization strategy and the source model emulate the properties of brain activity's actual generators. This added information is independent of both the recorded data and head model and suffices for obtaining a unique solution compatible with and aimed at analyzing experimental data. The inverse solution's features are evaluated with event-related potentials (ERPs) from a healthy subject performing a visuo-motor task. Two aspects are addressed: the concordance between available neurophysiological evidence and inverse solution results, and the functional localization provided by fMRI data from the same subject under identical experimental conditions. The localization results are spatially and temporally concordant with experimental evidence, and the areas detected as functionally activated in both imaging modalities are similar, providing indices of localization accuracy. We conclude that biophysically driven inverse solutions offer a novel and reliable possibility for studying brain function with the temporal resolution required to advance our understanding of the brain's functional networks.

(c) 2003 Elsevier Inc. All rights reserved.
\end{abstract}

Keywords: Inverse problem; Distributed source model; LAURA; ELECTRA; Regularization; Biophysical constraints

\section{Introduction}

The noninvasive three-dimensional reconstruction of the generators of the brain's electromagnetic activity measured at the scalp has been termed brain electromagnetic tomography (BET). In

* Corresponding author. Functional Brain Mapping Laboratory, Neurology Department, University Hospital of Geneva, 24 Rue Micheli du Crest, 1211 Geneva, Switzerland. Fax: +41-22-3728333.

E-mail address: Rolando.Grave@hcuge.ch

(R. Grave de Peralta Menendez).

Available online on ScienceDirect (www.sciencedirect.com.) similarity with anatomical (magnetic resonance imaging; MRI) and functional tomographies (positron emission tomography or PET, single photon emission computed tomography or SPECT, and functional MRI or fMRI), the construction of a brain electromagnetic tomography requires the solution of an inverse problem. However, this inverse problem lacks a unique solution. In spite of this serious difficulty, there is an active past and ongoing research on this field because of the extreme clinical and research importance of the problem.

A reliable electromagnetic three-dimensional tomography is, hitherto, the only possible approach for noninvasively studying a direct reflection of neuronal activity in human subjects with the temporal resolution required to trace the dynamic behavior of the human brain. In contrast to hemodynamic techniques, electrically reconstructed tomographic images are directly linked to neuronal processes. Because of their high temporal resolution, these images provide information about the short time lived neuronal networks subserving sensory and cognitive events.

Historically, the predominant approaches for solving the BET associated inverse problem operated on the assumption that only a discrete number of generators (usually dipoles) were active at a given time or over a period (e.g., Mosher et al., 1992; Murray et al., 2002; Scherg, 1990; Sekihara et al., 2002). However, since the functional activation images produced by these approaches cannot be considered tomographic reconstructions, they will not be considered in further detail here (though see Table 1).

A second family of distributed solutions to the BET uses the general theory developed for linear underdetermined inverse problems. Underdetermined means that the number of available measurements is smaller than the number of brain sites where the activity is sought after. This mathematical theory has been developed or extensively reviewed by Bertero et al. (1985), Groetch (1984), Parker (1994), and Tikhonov and Arsenin (1977) among others. Solutions to these problems are typically stated in terms of a so-called regularization operator (Tikhonov and Arsenin, 1977) fulfilling a double task: (1) picking one of the multiple possible solutions by introducing in the formulation of the problem some a priori information about the true solution and (2) providing stability to the solution, that is, small variations in the data should not lead to large variations in the source configuration.

So far the distributed solutions proposed to the BET inverse problem that opted for a regularization-based approach have 
Table 1

Major assumptions of commonly used methods for electromagnetic neuroimaging

\begin{tabular}{|c|c|c|c|}
\hline Inverse solution & Estimated field & Source model & $\begin{array}{l}\text { Regularization and/or } \\
\text { additional parameters }\end{array}$ \\
\hline ELECTRA + LAURA & LFPs (scalar) & Irrotational & $\begin{array}{l}\text { Mimics biophysical behavion } \\
\text { of irrotational (neural) fields }\end{array}$ \\
\hline Dipolar models & $\begin{array}{l}\text { Dipolar moments } \\
\text { (3D vector) }\end{array}$ & Irrotational & $\begin{array}{l}\text { Requires specification of the } \\
\text { number of sources }\end{array}$ \\
\hline $\begin{array}{l}\text { Minimum norm }(\mathrm{MN}) \\
\text { solution }\end{array}$ & Current density vector & $\begin{array}{l}\text { Irrotational sources } \\
\text { (ensured by the lead field) }\end{array}$ & Mathematically selected \\
\hline $\begin{array}{l}\text { L1 and L2 MN solutions } \\
\text { (weighted MN, minimum } \\
\text { Laplacian, etc.) }\end{array}$ & Current density vector & Unrestricted & Mathematically selected \\
\hline
\end{tabular}

largely relied on mathematically driven operators (see Grave de Peralta and Gonzalez, 1999 for several examples). While these operators could be reasonable for general academic problems, they lack a direct physiological or physical basis. This explains why the introduction of anatomically, physiologically, and functionally based a priori information is receiving increased attention (Bablioni et al., in press; Dale and Sereno, 1993; Fuchs et al., 1999; Hauk et al., 2002; Liu et al., 2002; Phillips et al., 2002).

This paper shows properties of neurophysiological generators that are specific to them and thus can be, but have not been, used as general constraints to the inverse problem. In particular, it is shown that neurophysiological currents are ohmic and can therefore be expressed as gradients of potential fields. This fact is used to reformulate the inverse problem in more restrictive terms, providing the basis for the noninvasive estimation of intracranial local field potentials (LFPs) from scalp recorded EEG data. The ohmic character of the currents is further used to pick a single solution to the inverse problem by imposing to the solution a spatial structure dictated by the physical laws that describe the propagation of electric potentials and fields in biophysical media. This paper gives a detailed derivation of these constraints together with a description of the steps required for their mathematical implementation. Aimed at a multidisciplinary readership, it combines the rigorous mathematical derivations with intuitive explanations about their physical or physiological meaning. The possibilities offered by this method to provide reasonable information about the spatio-temporal aspects of brain processing are illustrated in the analysis of ERPs recorded from a healthy subject performing a visuo-motor reaction time task for which fMRI results from the same experiment are available.

The paper is structured as follows. We first consider the principles leading from microscopic (neuronal level) to macroscopic measurements (LFPs) as well as the particular equation governing the electromagnetic fields and the quasi-stationary approximation. This section finishes with a mathematically oriented section devoted to the statement and solution of the inverse problem. The third section describes the constraints used for the source model (ELECTRA), as well as the method to obtain a unique solution (LAURA) based on constraints derived from the physiology and the physics of the problem. To this follows a fourth section describing the experimental design employed for both the fMRI and the ERP data experiment. The type of analysis performed for both kinds of data and the inverse solution results are presented in this section that also discusses the neurophysiological interpretation of the results in light of the available experimental evidence. A final general discussion focuses on providing the intuitive reasoning underlying the incorporation of biophysical constraints into the solution of the BET and its experimental support. The results obtained in the analysis of experimental data in this and previous papers that considered separately this source model or the regularization strategy are used to argue in favor of this type of solution. Future possible applications of this method are similarly introduced.

\section{Theory}

From the sources to the scalp fields

\section{Microscopic and macroscopic fields}

Brain function is investigated at two different scales: (1) A microscopic level encompassing the activity of a single or few neurons studied by single or multiunit recordings in animals and (2) A macroscopic level reflecting the activity of neuronal ensembles recorded by either intracranial LFPs in patients or animals or by scalp-recorded electric and magnetic fields.

At the origin of all these measurements are identical neural phenomena. During cell activation, large quantities of positive and negative ions cross the cell membrane, moving from the intracellular to the extracellular fluid, and vice versa. For all practical purposes, this ion movement is equivalent to a current flow, and it is responsible for all the recorded neurophysiological signals. The name used to refer to these microscopic currents varies somewhat. Within the modeling community, they are called impressed currents while most neurophysiological researchers term them active currents. Active or impressed are terms used to differentiate these currents from the passive (also termed return or volume) currents that manifest as the electrical response of the media to compensate for charge accumulation at specific sites driven by the active currents.

At the microscopic level the redistributions in extracellular ionic charge due to neuronal transmembrane current flows generate extracellular volume currents throughout the head. These microscopic volume currents, in turn set up field potential gradients that follow Ohm's law and are proportional both to the magnitude of the local currents and to the tissue conductivity. As such, they are termed ohmic currents.

For axonal and cardiac tissue several comparisons of the relative field strength from both impressed and volume currents at the microscopic level show only the latter to be significant (see Plonsey, 1982, and references therein). Consequently, at the macroscopic level observable by LFPs, electroencephalography (EEG), and magnetoencephalography (MEG), the primary currents are dominated by the microscopic volume currents and can therefore be modeled as ohmic currents. Macroscopic passive 
volume currents, result from the gross conductivity changes associated to the existence of different compartments in the head, that is, brain, cerebrospinal fluid, skull and scalp.

Importantly, since macroscopic primary sources are dominated by the microscopic volume currents, then the primary currents perceived by EEG and MEG are ohmic. The mathematical implication is that they can be modeled as irrotational currents (Grave de Peralta et al., 2001). As a consequence EEG and MEG measure essentially the same phenomena (Plonsey, 1982) and can both be described in terms of ohmic currents.

\section{Electromagnetic fields (Maxwell equations)}

The formal relationship between intracerebral currents and scalp-measured fields is expressed by Maxwell equations that describe the propagation of the electromagnetic fields within arbitrary volume conductor models, that is,

$$
\begin{aligned}
& \nabla \circ \boldsymbol{E}=\rho / \varepsilon \\
& \nabla \times \boldsymbol{E}=-\partial \boldsymbol{B} / \partial t \\
& \nabla \circ \boldsymbol{B}=0 \\
& \nabla \times \boldsymbol{B}=\mu(\boldsymbol{J}+\varepsilon \partial \boldsymbol{E} / \partial t)
\end{aligned}
$$

where $\boldsymbol{E}$ and $\boldsymbol{B}$ are the electric and magnetic fields, $\boldsymbol{J}$ is the total current density vector, $\varepsilon$ and $\mu$ stand for physical properties of the media, and $\rho$ is a (charge or current) density.

Eqs. (2') and (4') indicate that time varying electric and magnetic fields are interrelated. However, already in 1967, Plonsey and Heppner demonstrated that at the range of frequencies associated with electromagnetic fields in vivo-media (usually less than $1000 \mathrm{~Hz}$ ) it is possible to suppress the contribution of the temporal terms. This is called the quasistatic approach and implies that the capacitive and inductive effects produced by the temporal variations of the electric field $\boldsymbol{E}$ and the magnetic field $\boldsymbol{B}$ (see Eqs. (2') and (4')) are irrelevant. The practical consequence of the quasistatic approach is the assumption that electric and magnetic fields recorded at the scalp are the instantaneous reflection of the underlying neural processes. The electromagnetic processes taking place in the past are irrelevant for the present measurements. To date, no evidence against this approximation has been reported.

This quasistationary assumption allows for the separate modeling of the electromagnetic fields, that is, the electric field is not dependent upon temporal variations of the magnetic field and vice versa:

$$
\begin{aligned}
& \nabla \circ \boldsymbol{E}=\rho / \varepsilon \\
& \nabla \times \boldsymbol{E}=0 \Leftrightarrow \boldsymbol{E}=-\nabla V \\
& \nabla \circ \boldsymbol{B}=0 \Leftrightarrow \boldsymbol{B}=\nabla \times \boldsymbol{A} \\
& \nabla \times \boldsymbol{B}=\mu \boldsymbol{J} \Rightarrow \nabla \circ \boldsymbol{J}=0
\end{aligned}
$$

As described in the previous section, the total current emerging in biological tissue can be split into two terms, a primary neurophysiologically driven current $\left(\boldsymbol{J}^{\mathrm{p}}\right)$ and the volume or secondary current $\left(\sigma \boldsymbol{E}\right.$, i.e., $\left.\boldsymbol{J}=\boldsymbol{J}^{\mathrm{p}}+\sigma \boldsymbol{E}\right)$. Eq. (4) derives that the divergence of total current $(\boldsymbol{J})$ is zero, which combined with previous current decomposition, and Eq. (2) yields Poisson's equation for the electric potential field:

$\nabla \circ(\sigma \nabla V)=\nabla \circ \boldsymbol{J}^{\mathrm{p}}$

This equation establishes that the electric potential field, $V$, is generated by the divergence of the primary current, which reflects the fact that solenoidal (divergence-free) currents provide no contribution to voltage measurements (for a proof based on Green identities see Grave de Peralta et al., 2000). This theoretical result is the basis for the selection of the source space metric and the regularization operator described in next sections. According to this equation, plotting the modulus of the estimated primary current, which we would note has thus far been the common procedure used to depict inverse solutions results, does not reflect the actual generators. Instead, the actual generators are determined by the sources and the sinks obtained from the divergence of the primary current. This is mathematically identical to the Laplacian of the intracranial fields or the current source density (CSD).

In a similar way, assuming a divergence-free vector potential and substituting Eq. (3) in Eq. (4) we obtain the Poisson's equation for the magnetic field vector potential:

$\nabla^{2} \boldsymbol{A}=\mu \boldsymbol{J}$

In practice, Eqs. (5) and (6) refer to a given volume conductor model, e.g., multishell spherical models, etc., and should be solved together with some additional equations, called boundary conditions.

\section{The continuous and discrete inverse problem}

This section aims to formally describe the general mathematical formulation of linear inverse problems in a compact manner. The basic equations leading to the statement of the problem are given as well as the general solution for the underdetermined case. As such, the section is aimed to those interested in the practical implementation of the linear solution to the problem and can be skipped by nonmathematical readers. This section gathers information usually scattered through many different textbooks.

A compact formulation of the inverse problem can be given using the Green function formalism (Roach, 1970). Using Green functions, it is possible to express the inverse problem by a (first kind) Fredholm linear integral equation denoting the relationship between the data measured at the external point, $d(s)$, and the superposition of the contribution of the unknown current density vector at locations $r$ inside the brain (Fuchs et al., 1999; Greenblatt, 1993; Hämäläinen, 1993; Sarvas, 1987).

$d(s)=\int_{\text {Brain }} \boldsymbol{L}(s, r) \cdot \boldsymbol{J}(r) \mathrm{d} r$

The Green function is usually denoted in bioelectromagnetism as the scalar lead field and its gradient as the vector lead field (Malmivuo and Plonsey, 1995) or simply the lead field. Since the (vector) lead field function $\boldsymbol{L}(s, r)$ is the derivative of the Green function, it contains all the information about the boundary conditions as well as the media conductivities or permitivities for the electric and magnetic cases, respectively. In real conditions, neither the measurements nor the lead field functions are known for arbitrary surface and brain locations, but rather only at restricted 
a) $55-90 \mathrm{msecs}$

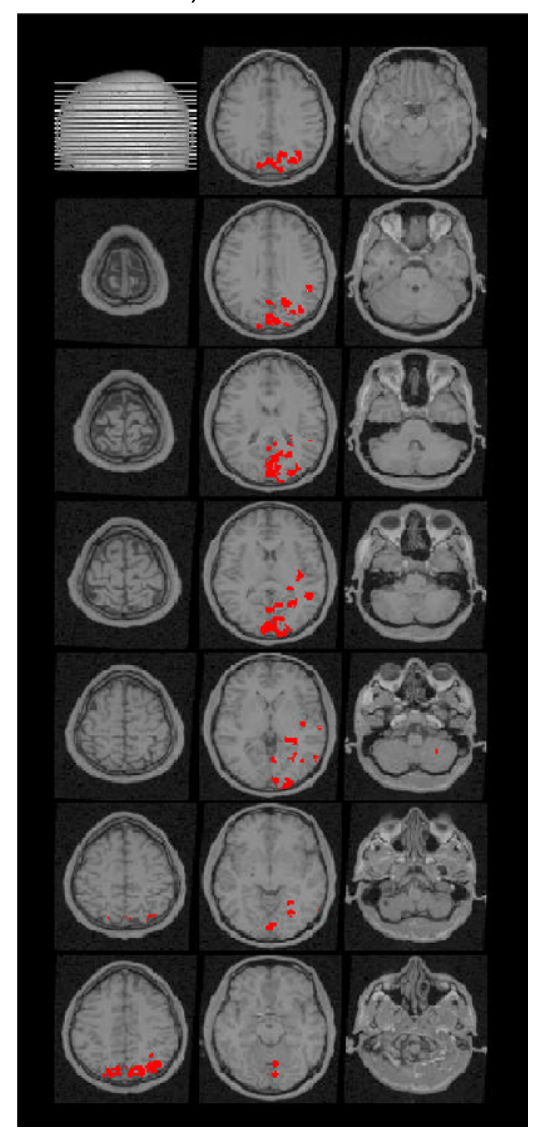

b) $100-200 \mathrm{msecs}$

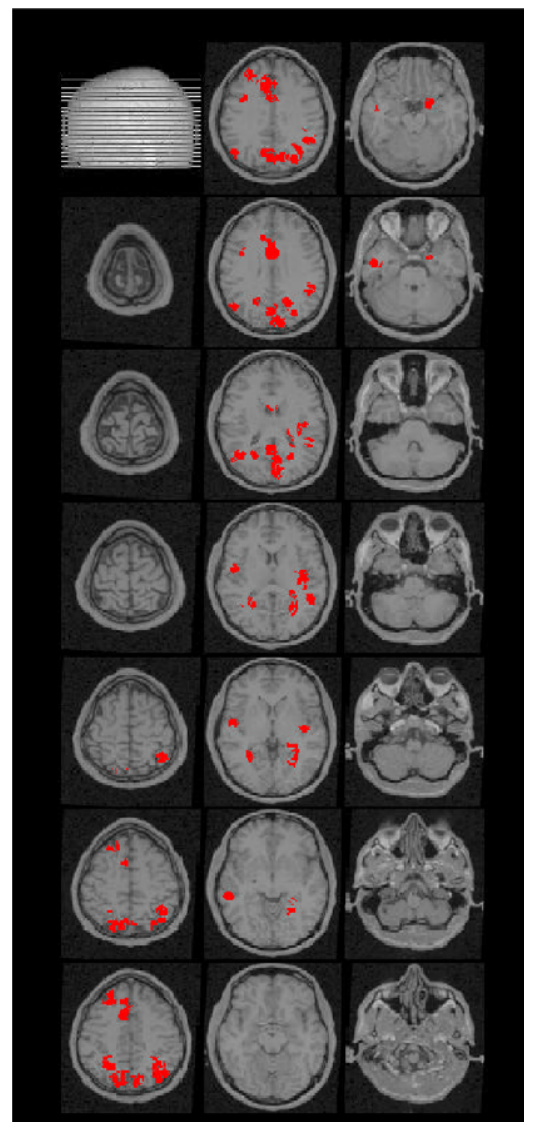

c) $220-276 \mathrm{msecs}$

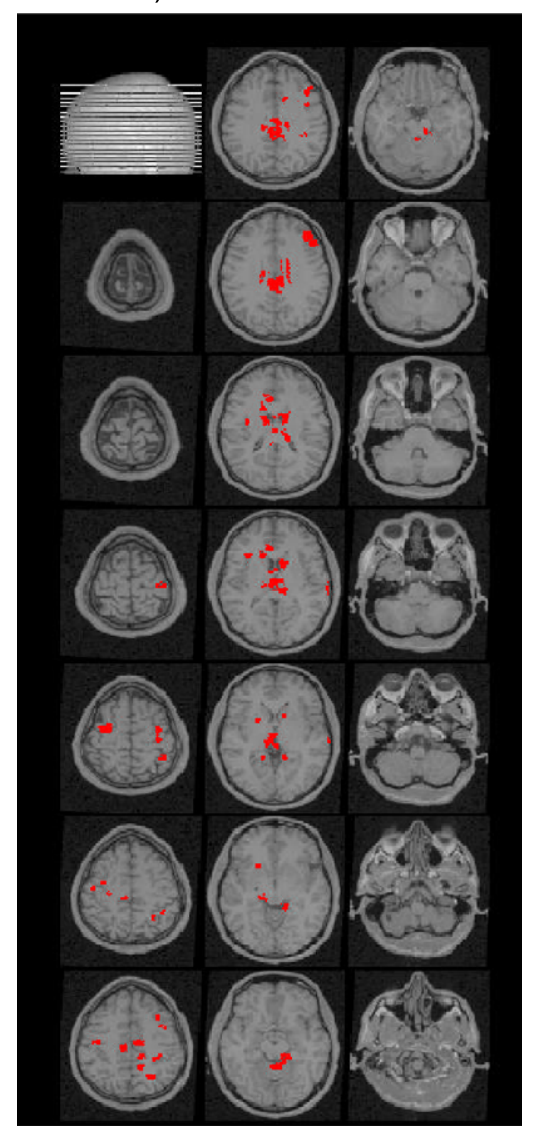

Fig. 1. Statistically significant electrical activation maps estimated by the inverse solution when a healthy subject performs a visuo-motor reaction time task. The statistical maps of activation are obtained by applying a $z$ test to the $z$ score transformed LFPs estimated using the inverse solution. Significance was set at $P<0.05$ after correcting for the number of independent tests. The three images correspond to averages over three different time periods were significant activation was observed: (a) 55-90 ms with activation at visual and parietal areas. (b) $100-200 \mathrm{~ms}$ and (c) $220-270 \mathrm{~ms}$. 


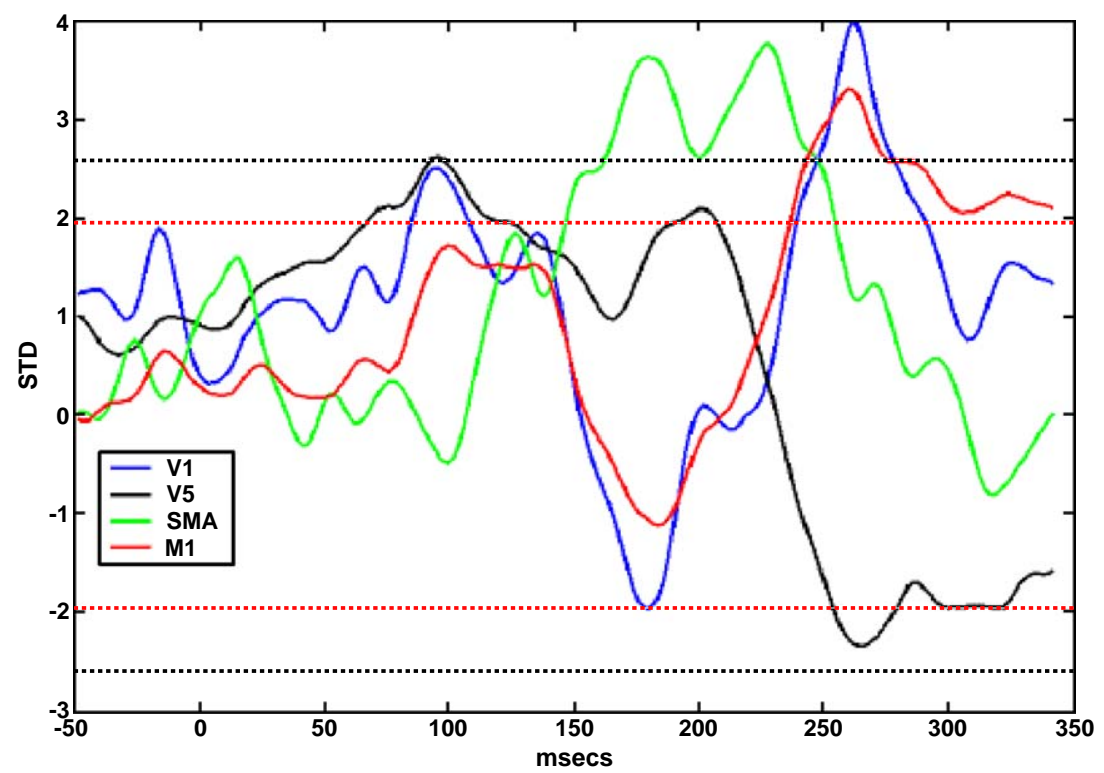

Fig. 2. Estimated LFPs transformed to $z$ score for two visual and two motor areas contralateral to the stimulated hemifield and responding hand : right SMA, right $\mathrm{M} 1$, right $\mathrm{V} 1$ and right $\mathrm{V} 5 / \mathrm{MT}$.

discrete sites. Thus, it is reasonable to introduce a discrete formalism where the integral equation in Eq. (7) is approximated by a discrete sum, which leads to the following underdetermined system of linear equations:

$\mathbf{d}=\mathbf{L} \mathbf{j}$

Vectors $\mathbf{d}$ and $\mathbf{j}$ and matrix $\mathbf{L}$ represent the discretization of the continuous functions, that is, $\mathbf{d}_{\mathrm{k}}=d\left(s_{\mathrm{k}}\right), \boldsymbol{j}_{\mathrm{m}}=\boldsymbol{J}\left(r_{\mathrm{m}}\right)$, and $\mathbf{L}_{\mathrm{km}}=\mathrm{w}_{\mathrm{km}}$ $\mathbf{L}\left(s_{\mathrm{k}}, r_{\mathrm{m}}\right)$ and $\mathbf{w}_{\mathrm{km}}$ are the quadrature weights.
The general solution of Eq. (8) can be obtained as the solution of the following variational problem (Grave de Peralta and Gonzalez, 1998; Menke, 1989):

$$
\min (\mathbf{L} \mathbf{j}-\mathbf{d})^{\mathrm{t}} \mathbf{W}_{\mathrm{d}}(\mathbf{L} \mathbf{j}-\mathbf{d})+\lambda^{2}\left(\mathbf{j}-\mathbf{j}_{\mathrm{p}}\right)^{\mathrm{t}} \mathbf{W}_{\mathrm{j}}\left(\mathbf{j}-\mathbf{j}_{\mathrm{p}}\right)
$$

Where $\mathbf{W}_{\mathrm{d}}$ and $\mathbf{W}_{\mathrm{j}}$ are symmetric (semi) positive definite matrices representing the (pseudo) metrics associated with the measurement space and the source space, respectively. Vector $\mathbf{j}_{\mathrm{p}}$ denotes any available a priori value of the unknown, e.g., from other varieties of brain functional images. The regularization parameter is denoted

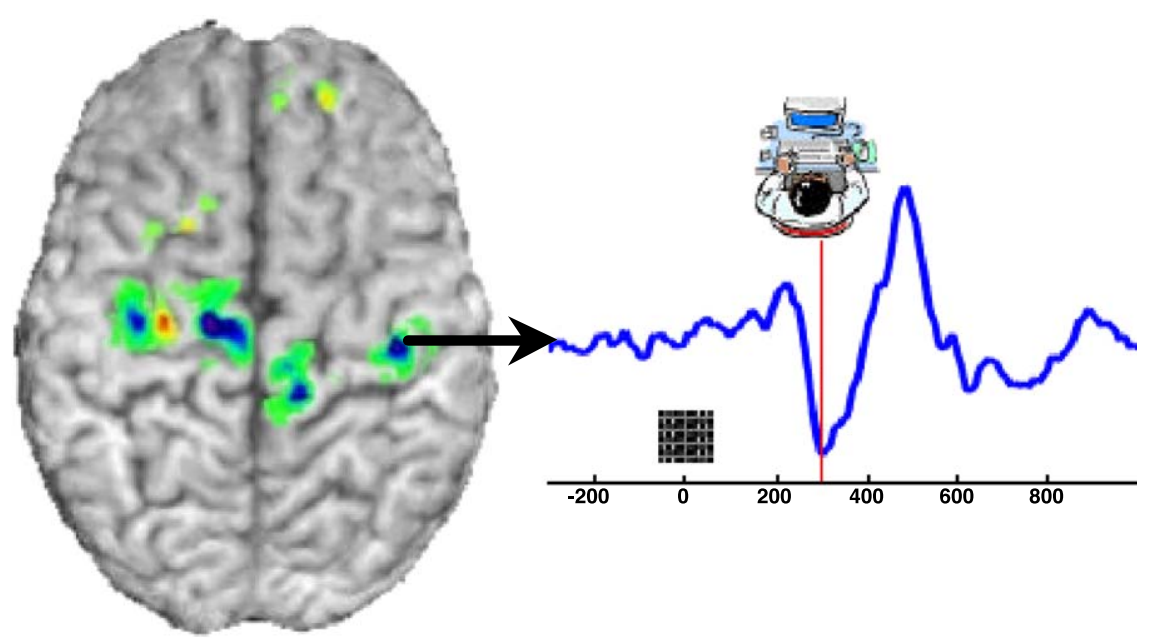

Fig. 3. Estimation of LFPs using a biophysically constrained linear inverse solution (ELECTRA source model and LAURA regularization strategy). The spatial distribution of LFP at mean reaction time $(300 \mathrm{~ms})$ is shown at the left part of the figure as an example of the spatial resolution provided by the method. The temporal behavior of the LFP at the probable location of the right M1 is shown at the right. The red vertical line marks the mean reaction time for the subject for whom the spatial map at the left is shown. This is the raw image produced by the inverse solution without any statistical preprocessing. Note the strong negativity shown by the LFP at M1 accompanying the motor response that resembles the behavior of LFP recorded in monkeys when performing hand movements. 
by $\lambda$. Independently of the rank of $\mathbf{L}$, the solution to Eq. (9) is unique if and only if the null spaces of $\mathbf{W}_{\mathrm{j}}$ and $\mathbf{L}^{\mathrm{t}} \mathbf{W}_{\mathrm{d}} \mathbf{L}$ intersect trivially, that is, $\operatorname{Ker}\left(\mathbf{W}_{\mathrm{j}}\right) \cap \operatorname{Ker}\left(\mathbf{L}^{\mathrm{t}} \mathbf{W}_{\mathrm{d}} \mathbf{L}\right)=\{0\}$. In this case, the estimated solution vector $\hat{\mathbf{j}}$ can be obtained using the change of variable $\mathbf{j}=\mathbf{j}_{\mathrm{p}}+h$ and solving the resulting problem for $h$, that is:

$\hat{\mathbf{j}}=\mathbf{j}_{\mathrm{p}}+\left[\mathbf{L}^{\mathrm{t}} \mathbf{W}_{\mathrm{d}} \mathbf{L}+\lambda^{2} \mathbf{W}_{\mathrm{j}}\right]^{-1} \boldsymbol{L}^{\mathrm{t}} \mathbf{W}_{\mathrm{d}}\left[\mathbf{d}-\mathbf{L j}_{\mathrm{p}}\right]$

If and only if matrices $\mathbf{W}_{\mathrm{j}}$ and $\mathbf{W}_{\mathrm{d}}$ are positive definite, Eq. (10) is equivalent to:

$\hat{\mathbf{j}}=\mathbf{j}_{\mathrm{p}}+\mathbf{W}_{\mathrm{j}}^{-1} \mathbf{L}^{\mathrm{t}}\left[\mathbf{L} \mathbf{W}_{\mathrm{j}}^{-1} \mathbf{L}^{\mathrm{t}}+\lambda^{2} \mathbf{W}_{\mathrm{d}}^{-1}\right]^{-1}\left[\mathbf{d}-\mathbf{L} \mathbf{j}_{\mathrm{p}}\right]$

In the case of null a priori estimates of the current distribution $\left(\mathbf{j}_{\mathrm{p}}=0\right)$ and perfectly accurate data, that is, approaching zero, the general solution can be written as (Rao and Mitra, 1971, sect. 3.3.3):

$\hat{\mathbf{j}}=\mathbf{G d} \quad$ with $\mathbf{G}=\mathbf{W}_{\mathrm{j}}^{-1} \mathbf{L}^{\mathrm{t}} \mathbf{W}_{\mathrm{d}} \mathbf{L}\left(\mathbf{L}^{\mathrm{t}} \mathbf{W}_{\mathrm{d}} \mathbf{L} \mathbf{W}_{\mathrm{j}}^{-1} \mathbf{L}^{\mathrm{t}} \mathbf{W}_{\mathrm{d}} \mathbf{L}\right)^{-} \mathbf{L}^{\mathrm{t}} \mathbf{W}_{\mathrm{d}}$

If $\mathbf{L} \mathbf{W}_{\mathrm{j}}^{-1} \mathbf{L}^{\mathrm{t}}$ is invertible we can take the limit of expression 11 (with respect to $\lambda$ ) to obtain a simpler expression, that is:

$\mathbf{G}=\mathbf{W}_{\mathrm{j}}^{-1} \mathbf{L}^{\mathrm{t}}\left(\mathbf{L} \mathbf{W}_{\mathrm{j}}^{-1} \mathbf{L}^{\mathrm{t}}\right)^{-1} \mathbf{L}^{\mathrm{t}} \mathbf{W}_{\mathrm{d}}$

Inverse matrix $\mathbf{G}$ is the unique generalized inverse (Ben-Israel and Greville, 1974, chap. 2, sect. 5) with kernel and range defined by kernel $(\mathbf{G})=\mathbf{W}_{\mathrm{d}}^{-1} \boldsymbol{M}(\boldsymbol{L})^{\perp}$ and range $(\mathbf{G})=\mathbf{W}_{\mathrm{j}}^{-1} \boldsymbol{M}\left(\boldsymbol{L}^{\mathrm{t}}\right)$ where $\boldsymbol{M}(\boldsymbol{L})=\operatorname{range}(\boldsymbol{L})$ denotes the space spanned by the columns of $\boldsymbol{L}$ and the superscript $\perp$ stands for the orthogonal complement. This relationship sheds light on the dependence that exists between the selected metrics and the only kind of sources that can be perfectly retrieved that is, those that belong to range $(\mathbf{G})$. To see that the variational approach includes all linear inverse solutions, note that for any arbitrary inverse matrix $\mathbf{G}$ there are (at least) a pair of metrics $\mathbf{W}_{\mathrm{j}}$ and $\mathbf{W}_{\mathrm{d}}$ that, when optimized in the sense of Eq. (9), produce the desired inverse. For example, the minimum norm solution (Hämäläinen and Ilmoniemi, 1984; Penrose, 1955; Rao and Mitra, 1971) is obtained when both metrics are the identity matrix.

\section{Biophysical Constraints to solve the inverse problem}

The previous section discussed the general mathematical formalism for solving linear inverse problems. However, their solution can be drastically improved by considering the physical or technical details concerning the particular inverse problem we want to solve. Consequently, in this section we consider the inclusion of a priori information derived from the biophysical laws characterizing the generation and propagation of electromagnetic fields in volume conductor media. In particular, we will discuss two types of biophysical constraints, one related to the source model and a second one concerning the selection of a regularization strategy. We would note that both constraints are independent of the experimenter; a point to which we will return in our discussion of the present approach in comparison with those at present typically used by the neuroscientific community.

\section{Biophysical constrains on the Source Model: ELECTRA}

As described in Eq. (7), the inverse problem can be compactly represented by the Green function $\psi$ that includes all the boundary conditions. Using that formalism, Eq. (5) can be rewritten as:

$$
\begin{aligned}
V(r) & =\int_{\text {brain }} \nabla \circ \boldsymbol{J}\left(r^{\prime}\right) \psi\left(r, r^{\prime}\right) \mathrm{d} r^{\prime}=\int_{\text {brain }} \nabla \varphi\left(r^{\prime}\right) \circ \nabla \psi\left(r, r^{\prime}\right) \mathrm{d} r^{\prime} \\
& =\int_{\text {brain }} \nabla \varphi\left(r^{\prime}\right) \circ \boldsymbol{L}\left(r, r^{\prime}\right) \mathrm{d} r^{\prime}
\end{aligned}
$$

The term $\nabla \circ \boldsymbol{J}^{\mathrm{p}}\left(r^{\prime}\right)=I\left(r^{\prime}\right)$ is usually referred as the CSD (e.g., Mitzdorf and Singer, 1977) and $\boldsymbol{L}\left(r, r^{\prime}\right)=\nabla \psi\left(r, r^{\prime}\right)$ is the vector lead field.

Eq. (14) confirms that the potential measured at the scalp is produced by the divergence of the primary sources. It means that divergence-free vector fields produce no measurable potentials. For this reason we proposed in Grave de Peralta et al. (2000) to consider irrotational sources as the only responsible for the observed EEG data. An example of irrotational source model is the current dipole commonly used to model neuronal sources. The proof that the dipole is an irrational source is given in Appendix A.

The source model denoted by ELECTRA can be equivalently solved for three different physical magnitudes, consistent with the source model (irrotational currents): (1) Estimation of an irrotational current density vector $\boldsymbol{J}^{p}=\nabla \varphi$ with the vector lead field $\nabla \psi$. (2) Estimation of a scalar field, the CSD, $\nabla \circ J^{\mathrm{p}}\left(r^{\prime}\right)=I\left(r^{\prime}\right)$ with the scalar lead field $\psi$. (3) Estimation of a scalar field, the potential distribution $\varphi$ in $Q$ with a transformed scalar lead field $\nabla \psi\left(r, r^{\prime}\right) \circ \nabla$.

ELECTRA can be intuitively described as the noninvasive estimation of LFPs by virtual intracranial electrodes. Consequently, the instantaneous estimates of intracranial source activity produced by ELECTRA substantially differ from those produced by typically used approaches that assume the current density vector as the source model. The major difference in terms of the instantaneous maps is that ELECTRA calculates a scalar field and leads to reconstructions of LFPs with both positive and negative values. In contrast, typical approaches depict the modulus of the current density vector at each solution knot (voxel), which is always a positive value. Thus, in addition to the theoretical advantage of these biophysical constraints, there is in parallel a mathematical advantage in that the number of unknowns estimated by the inverse model is threefold fewer. Moreover, the LFPs derived by ELECTRA provide polarity information, which is useful experimentally particularly for comparison with intracranial recordings in humans (e.g., Thut et al., 2000) and animals. In more practical terms, standard methods often propose one extended source in many situations, whereas ELECTRA proposes two sources with equal or different polarities. These elements therefore make difficult (on both mathematical as well as interpretational levels) a straightforward comparison of results provided by this method with previously published inverse solutions. Nonetheless, we here include a tabulation of the central aspects of different families of inverse solution methods used by the neuroscientific community (see Table 1). We do this to provide the reader with an overview of the major assumptions determining the strengths and limitations of each approach. Even though this restriction of the ELECTRA source model reduces the degree of 
underdeterminancy of the inverse problem, it is not sufficient to produce a unique solution. However, the next section considers physically sound a priori information that can be used to restore uniqueness to bio-electromagnetic inverse problems.

\section{Constraints on the regularization strategy: LAURA}

The goal of this section is to illustrate that in the bioelectric inverse problem a sound selection of the regularization operator can be derived from biophysical laws. This information can be included in the estimation procedure to pick a solution fulfilling both the observed data and the bio-electromagnetic constraints. What will be sustained throughout this section is that neurophysiological currents create electromagnetic fields and potentials that extend beyond the spatial location where they are generated and flow into the space that surround them. The strength of the potentials or fields at a certain brain location depends upon the strength of the current creating the field and upon a power of the distance separating the site where the current is created to the place where its effect is detected. This is precisely the idea behind the regularization strategy termed LAURA (Local Auto-Regressive Averages) and described in Grave de Peralta et al. (2001) and Grave de Peralta and Gonzalez (2002). In this approach the relationship between the brain activity at one point and its neighbors is expressed in terms of a local autoregressive estimator with coefficients depending upon a power of the distance from the point. The details of this regularization strategy are described in Appendix B.

The particular selection of the power used for the distance is determined by two factors: (1) the source model we consider as more likely to emulate actual brain generators and (2) the type of unknowns we are estimating, that is, LFPs or current density vectors. For instance, a monopolar source will produce a field that decreases with the distance more slowly than the field of a dipolar source. On the other hand, electric fields decay faster than potential fields. The optimal biophysical selection should be based on actual measurements of the speed of decay of LFPs with the distance to their generators in humans or animals. As an example, we will assume in what follows a widely used model of ohmic current, the current dipole (see Appendix A), as the basis to derive the relationships to be used for the estimation of LFPs and current density vectors.

Let's start by considering the estimation of LFPs in depth, described in the previous section as the variant (3) of ELECTRA source model. The potential field measured at a point $r$ due to a current dipole (irrotational source) at point $r^{\prime}$ with dipolar moment $M$ is given by:

$\phi(r)=M \frac{r-r^{\prime}}{\left|r-r^{\prime}\right|^{3}}=\frac{|M| \cos \theta}{\left|r-r^{\prime}\right|^{2}}$

where $\theta$ is the angle between the dipole moment and the $r-r^{\prime}$ vector. This equation expresses that, according to electromagnetic theory, LFPs at a given point depend upon the activity at another brain site according to a square inverse law. It thus suggests that while estimating LFPs, the activity at neighbor points should be related by the inverse of the squared distance.

For the case of the estimation of the vector field $\boldsymbol{J}$, that is, the current density vector, we can take into account the ohmic character of the measurable primary currents to express the unknowns in terms of the electric field produced by a dipolar source, that is,

$\boldsymbol{J}^{\mathrm{p}}(r)=\sigma \boldsymbol{E}=\frac{1}{4 \pi} \frac{3(M \hat{r}) \hat{r}-M}{|r|^{3}}$

Where $\hat{r}$ denotes a unit vector extending from the center of the dipole to the point where we compute the field. This equation expresses that the strength of electric fields (and accordingly ohmic currents generated by dipolar sources) fall off with the inverse of the cubic distance to the target point, and thus provides a physically sound argument to select the exponents when dealing with the estimation of the current density vector. It also expresses that a particular Cartesian component of the current at a given site depends upon all the components of the field. We could then either apply the same regularization operator to each individual Cartesian component of the primary current density vector (3D vector field) or incorporate the dependencies between the dipole moments into the model. Introduction of explicit dependences among the current density components as described by Eq. (16), is another clear example of sensible physical constraints that can be used in solving the bioelectromagnetic inverse problem.

All these biophysical elements allow for the selection of a sound metric to be used as a regularization operator as shown in detail in Appendix B.

\section{Data analysis}

The previous section describes the approach to obtain a unique solution to the bioelectric inverse problem that relies on physically derived a priori information about the generators and the fields they produce in biological media. As any solution to underdetermined inverse problem, this solution should provide accurate results if the actual generators fulfill the properties we are incorporating as a priori information and is likely to fail otherwise. In principle, the best manner to assess the results of the inverse problem would be to compare them with intracranial recordings from the same subjects. Unfortunately, simultaneous high-density scalp recordings and intracranial recordings on the same subject are seldom available and are not hitherto at our disposal.

Testing a biophysically designed inverse solution with artificial probe sources (dipoles or monopoles) will not evaluate the capabilities of the solution to deal with experimental data. We have provided evidence that experimental data are generated by ohmic currents, a fact that establishes fixed spatial dependencies between the activity (potential and/or current) at a brain site and activity at all other sites. These relations are absent in the currents attributed to the type of probe sources commonly used to evaluate distributed inverse solutions in the field. Such probe sources are constructed with a value of one for a Cartesian component at a single node and zero elsewhere. For this reason, we prefer to report the results of applying the inverse solution described here to the analysis of averaged ERP data recorded from a single healthy subject performing a visuomotor reaction time task. The results are discussed in the framework of the experimental evidence for similar tasks available from independent imaging modalities and intracranial recordings in animals. The fMRI results of the same subject under an identical experimental paradigm are presented as an independent manner to assess the localization capabilities of the solution. 

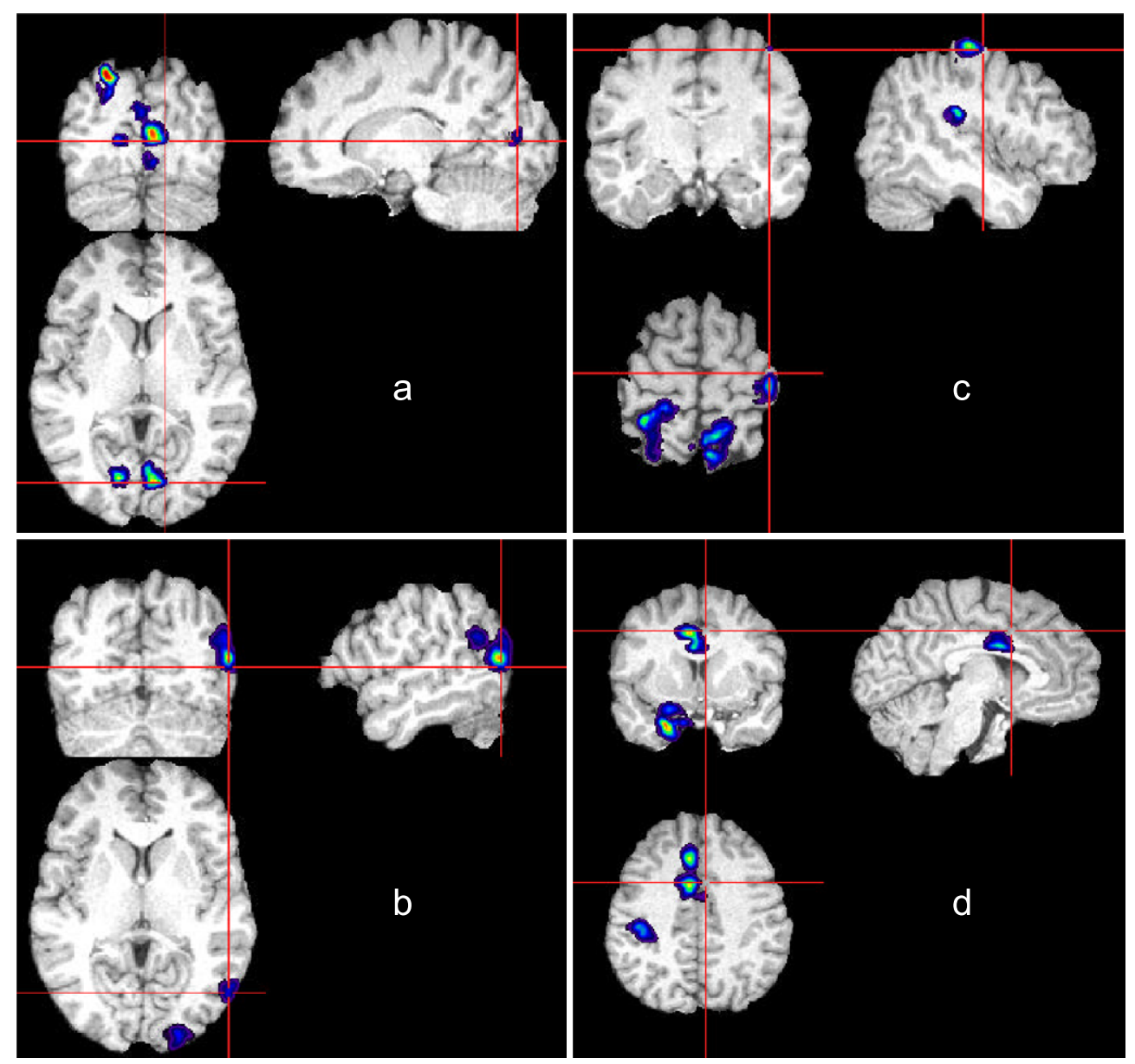

Fig. 4. Inverse solution and fMRI results for the same areas shown in Fig. 2 overlaid onto the high-resolution anatomical image of the experimental subject. The results of the statistical analysis of the inverse solution for: (a) right V1, (b) right V5/MT, (c) right M1 and (d) SMA are shown. The empty space at the intersection of the red crosshair marks the center of activation detected by the fMRI. Axial, coronal and transverse views are shown for each area. Each inset is presented at the time of maximal activation of the corresponding area.

\section{Experimental protocol and recording methods}

\section{ERP and fMRI experimental paradigm}

A healthy young subject (male, 30 years) completed a simple reaction time task while fixating a central cross, as white and black checkerboard stimuli appeared in either the left or right visual field. The subject was asked to press a sequence of four keys with his left hand upon stimulus detection in either visual field. Reaction time was measured according to the first key pressed. Stimuli were centered at $9.5^{\circ}$ eccentricity, measured $3^{\circ}$ wide $\times 4^{\circ}$ high, and appeared with equal probability in each visual field. Stimuli were presented for $100 \mathrm{~ms}$ duration. Only stimuli presented to the left visual field were considered in the present analyses. In the EEG portion the ISI was randomised between 1500 and $2500 \mathrm{~ms}$, and stimuli were blocked into series of 120 trials. The subject completed four blocks of trials after acclimating to the task during a practice session. In the event-related fMRI protocol, the interstimulus interval varied between 14.125 and $17.875 \mathrm{~s}$, and the number of trials was 64 .

\section{ERP recording and analysis}

The EEG was recorded at $500 \mathrm{~Hz}$ from 125 scalp-electrodes (Electric Geodesic Inc. system). Head position was stabilized with a head and chin rest. Off-line processing of the scalp data consisted of visual rejection of trials contaminated by artefacts and interpolation of bad channels. The average ERP (300 ms prestimulus to $600 \mathrm{~ms}$ poststimulus onset) was computed based on the 126 trials that remained after artefact removal and were recalculated against the average reference.

\section{FMRI recording}

Functional images were obtained using a single shot gradientecho EPI sequence (TR $=2 \mathrm{~s}, \mathrm{TE}=60 \mathrm{~ms}, \mathrm{FoV}=240 \mathrm{~mm}$, matrix size $64 \times 64)$, on a $1.5 \mathrm{~T}$ Siemens Magnetom Vision. Each volume was made of 16 images parallel to the bicommissural plane covering the entire brain (slice thickness $5 \mathrm{~mm}$, gap $1 \mathrm{~mm}$ ). The acquisition was made of 512 volumes.

\section{Data analysis}

\section{Functional magnetic resonance data analysis}

The results of the fMRI recording were analyzed using SPM99. Data were first spatially and temporarily realigned, normalized to a standard brain based on the MNI (Montreal Neurological Institute) template, and smoothed with an isotropic Gaussian kernel (FWHM = $9 \mathrm{~mm})$. The statistical analysis was carried out according to the General Linear Model, using as basis functions the canonical 
hemodynamic response function and its temporal derivative. An $F$ test was performed to obtain the statistical parametric map that was thresholded at $P<10-6$ (nonadjusted) to identify the active voxels.

\section{Inverse solution computation and temporal analysis}

A realistic lead field model was computed based on the subject's anatomical MRI. Conductivity values for the three basic layers (scalp, skull and brain) were selected as in Stock (1987). The solution space was formed using 3255 pixels distributed within the gray matter, providing a spatial resolution of $7 \mathrm{~mm}$ in the saggital and coronal directions and $6 \mathrm{~mm}$ in the transverse direction. The LFPs at each solution node were computed for the average ERP using variant (3) of ELECTRA source model and LAURA's based regularization (see Appendix B for details on the implementation). The exponent used to weight the distance discussed in the previous section and the appendix was set to two (the proposed selection for estimation of potentials due to dipoles) and the weights set to one.

Each LFP at a single knot was transformed to $z$ score considering the mean and standard deviation of the whole ERP interval that included $300 \mathrm{~ms}$ of prestimulus. This procedure aimed to select pixels with consistent responses to the stimuli during the whole (poststimulus) period to allow for a simpler comparison with the single fMRI statistical image associated to the whole interval. A $z$ test was applied to the $z$ score results $(P<0.05)$ after correcting for the number of independent tests (see Appendix $C$ ).

\section{Results}

\section{Inverse solution activation}

Statistical analysis of the estimated LFP yields a clear temporal separation of three response periods (Fig. 1). A first period (approximately 55-90 ms poststimulus) showed focal activation at ipsilateral visual areas as well as bilateral superior parietal areas and precuneus (Fig. 1a). Significant activation was also observed at the right infero-temporal gyrus and right insula. During a second period lasting from 100 to $200 \mathrm{~ms}$ (Fig. 1b) we observed spreading of activity to contralateral visual areas of both hemispheres as well as dispersal of activity over superior parietal and parieto-occipital areas. Supplementary motor cortex activation started during this period. A last period appeared around $220 \mathrm{~ms}$ lasting up to $276 \mathrm{~ms}$, that is, $30 \mathrm{~ms}$ before mean reaction time $(300 \mathrm{~ms})$ and basically comprised bilateral motor and premotor areas, occipito-parietal areas, bilateral activation of the parietal cortex and temporal lobe (Fig. 1c). At the time of the response (not shown) or slightly after it, we found significant activation at the ipsilateral cerebellum, contralateral somatosensory areas, SMA, contralateral putamenpallidum, right frontal lobe and at contralateral visual, motor and premotor areas.

The temporal analysis of the inverse results represented in Fig. 2 for SMA, V1, V5 and M1, indicates a very early and nearly simultaneous activation of visual areas V1 and MT. The onset of activation occurs near $50 \mathrm{~ms}$ after presentation of the visual stimuli. This early activation coincides with independent studies on the visual system in humans (e.g., Buchner et al., 1997) and animals (e.g., Schroeder et al., 1998) that might be explained by parallel input to both visual areas (Ffytche et al., 1995). Activation of superior parietal areas was also very early, occurring within the first 90-ms poststimulus, which suggest a fast pathway for visuo- motor transformation via the dorsal visual processing stream (e.g., Murray et al., 2001).

Fig. 3 provides an example of the localization results and spatial resolution provided by the inverse solution. It represents the average over the $40 \mathrm{~ms}$ preceding the motor response of the solution overlaid onto the individual subject brain using the MRICro software (Rorden and Brett, 2000). The temporal response of the contralateral motor area M1 is also shown. The similarity between the estimated LFP in humans and that recorded with intracranial electrodes over the primary motor cortex in monkeys (see Fig. 1B in Donchin et al., 2001) around the time of hand movement is remarkable. A strong negative deflection is seen to occur at the time of the movement. Such negative deflections are usually interpreted as reflecting excitatory spike-causing input to neurons in the neighborhood of the electrode (Arieli et al., 1995). A positive deflection is observed after movement execution that exactly coincides with the recordings on the monkeys.

fMRI activation revealed major clusters in bilateral V1, V5, and primary motor areas. Strong SMA activation was also observed. Further clusters of activation appeared at the superior parietal lobe and basal ganglia. Producing a summarized image over time of the inverse solution results and the fMRI results revealed strong similarities in terms of the functionally active areas, as well as some small differences. For instance, inverse solution showed activation at the temporal lobes that did not appear on the functional images. Still correspondence between both modalities is rather good and localization results for the major activated areas were excellent. Some examples of this correspondence are shown in Fig. 4 that depicts the inverse localization results and the fMRI main activation centroids both overlaid onto the individual subject MRI. The red crosses indicate the fMRI activation centroids for the ipsilateral V5, M1, V1 and SMA. The intensity map reflects the inverse solution results.

\section{Discussion}

In the preceding sections, we described properties of biophysical generators that can be used to single out a unique solution to the bioelectromagnetic inverse problem. In particular, we showed that existing experimental evidence supports that ohmic currents produce both scalp and intracranial LFPs. The ohmic character of the currents is here used to derive a formulation of the inverse problem (ELECTRA) that aims to noninvasively estimate intracranial LFPs from scalp-recorded data. This formulation reduces by a factor of three the number of unknowns to be estimated from the same amount of data, leading to a less underdetermined inverse problem. A unique solution to the inverse problem is obtained using a regularization strategy (LAURA) that imposes a spatial structure to the solution derived from electromagnetic laws. This unique solution is therefore conceived to be optimal in the sense of resembling actual brain generators. Importantly, the combination of LAURA's metric with ELECTRA's source model produces a linear distributed inverse solution that differs from the one obtained using ELECTRA source model and Tikhonov regularization (Grave de Peralta et al., 2000) or from a solution combining LAURA's regularization and a source model based on the estimation of the current density vector (Grave de Peralta et al., 2001; Grave de Peralta and Gonzalez, 2002). By way of example (and to clarify this point), we would note that both the Minimum Norm (MN) and Weighted Minimum Norm (WMN) methods use the same source 
model, though with different regularization operators, which in turn leads to different results. In the same manner, ELECTRA source model with Tikhonov regularization produces a result that is different from the one obtained with the same ELECTRA source model and LAURA's regularization approach. Thus, the combination of ELECTRA and LAURA presented here, indeed constitutes a new inverse solution method.

Theory and practice in solving underdetermined inverse problem indicate that the only way to overcome lack of information is by completing the data with expected features of the unknowns we seek to estimate. However, the information (a prioris) added should be independent of, that is, not reflected in the model (the lead field), and complementary to the data. The spatial structure imposed by LAURA regularization strategy is not included in the model because lead field computations rely upon the use of currents produced by dipolar probe sources at each solution knot (one at the solution knot and zero elsewhere). Thus incorporating into the constraints to the solution the spatial relationship between LFP or electric fields dictated by biophysical laws, results in additional and independent a priori information that suffices to guarantee a unique solution to the problem.

This novel combination of the ELECTRA source model with the LAURA regularization operator is distinct from currently used approaches in several significant ways (see Table 1 for summary). While the irrotational character of bioelectric sources is indeed appropriately incorporated in dipolar models, such methods rely on severe user-dependent a priori information (e.g., the number of sources to be estimated). In contrast, distributed inverse solution methods do not have such an assumption regarding the number of active sources. However, they are in general unable to ensure that the estimated sources are indeed irrotational. The use of a lead field computed from dipolar sources, that is, irrotational sources, is insufficient to ensure the irrotationality of the results, except for the minimum norm solution that has no component on the null space. For any other distributed linear solution with a nontrivial component on the null space, there is no way to guarantee that estimates are irrotational unless this is explicitly incorporated as a constraint (see Table 1 for further comparison). For that reason an explicit implementation of the irrotationality constraint is required.

The remaining question is the evaluation of the physiological reliability of the incorporated a prioris, that is, do LFPs actually behave according to the constraints we have incorporated into the solution? While there is no doubt about the ohmic character of the primary currents, further experimental research using intracranial recordings might be required to assess the exponent used in LAURA's regularization approach to model the speed of decay of electric potentials with the distance to the source.

In previous studies (Grave de Peralta and Gonzalez, 2002 and Grave de Peralta et al., 2001) we have reported the capabilities of LAURA's regularization strategy to localize single probe sources and compared its results with those of the most widely used source localization methods. According to these results the use of high exponent values, that is, fields that decay very fast with the distance to the source, yields to significant improvements of the single source localization with respect to other previously tested solutions (Grave de Peralta et al., 2001). However, it is not our goal to localize with distributed solutions artificial probe sources, but rather experimental data. Besides, evaluating the behavior of the solution with probe sources provides no information about the behavior of the same solution when multiple sources are simultaneously active (Grave de Peralta and Gonzalez, 1998). Previous arguments indicate that inverse solutions designed to localize biophysical generators should be evaluated with experimental data or with independent and well assessed techniques used for functional localization.

We have evaluated the estimation of intracranial potentials using the ELECTRA-approach by direct comparison with intracranial recordings in epileptic patients. In Michel et al. (1999) we validated this technique in the localization and study of propagation of interictal activity In another study, visual activity in the motor cortex had been proposed from the analysis of surface evoked potentials in healthy subjects and was then confirmed with intracranial recordings in patients (Thut et al., 2000). Finally, fast activation of extrastriate visual areas in color-coded motion stimuli have been described on the basis of the estimated potentials (Morand et al., 2000). Concerning the LAURA regularization strategy, meaningful results have been obtained in studies on multisensory memory processes (Murray et al., in press), auditory recognition (Ducommun et al., 2002), illusory contour perception (Pegna et al., 2002), semantic processing (Khateb et al., 2003), as well as visual motion processing, mental imagery, face recognition, and semantic decision (Michel et al., 2001; Michel et al., 2003).

One of the aspects scarcely considered in the evaluation of inverse reconstructions is the quality of the estimated waveshapes. Relatively few studies have considered this problem on the framework of distributed models although with very interesting conclusions. The temporal reconstructions provided by linear L2-based distributed inverse solutions are better than those of spatiotemporal models (Schwartz et al., 2001) or L1-based reconstructions (Uutela et al., 1999). A few comparisons with intracranial data (Thut et al., 2000; Dale et al., 2000) are also extremely appealing, suggesting systematically that temporal reconstructions of the generators might be more reliable than their spatial counterparts. We believe that this observation could constitute the basis for considerable improvements of the spatial estimates. This is the idea behind transforming the estimated LFP to $z$ scores that serves not only to statistically assess the activated pixels, but results also in a powerful strategy to alleviate the pitfalls of the inverse solution. In fact, the inverse solution maps for a given time frame differ considerably from the functional activation maps obtained after the statistical transformation of the data. Spurious activity is minimized after this procedure because spurious activity tends to systematically appear over time leading to temporal traces of high amplitude but large temporal variance. Also the underestimation of the source amplitudes normally associated to deeper sources is palliated since each temporal trace is normalized with respect to itself. Consequently, a given pixel will be detected as functionally active at a given time if and only if its estimated LFP significantly exceeds at this time its mean estimated activity.

The accurate estimation of the temporal aspects of the LFPs is essential for the application of the proposed method to the study of the dynamics of brain function. For instance, noninvasive estimates of LFP could clarify the role of brain oscillations in the functioning of the human brain by assigning particular brain rhythms to specific areas. Another problem that could be directly addressed by this method is the investigation of a relationship between the BOLD hemodynamic response and LFPs. The existence of such relationships already assessed by Logothetis et al. (2001), in monkeys was found to be stronger for the high frequency part of the LFP. The technique proposed in this paper offers the possibility to continue this line of research noninvasively in a variety of experiments in humans. 
Although described and illustrated here for the analysis of electric data, the same model of ohmic currents could be used for the case of magnetic recordings. This will produce advantages, which are in mathematical terms similar to the ones described for the electric case.

\section{Conclusions}

In this paper we described properties of neural generators that can be used to obtain a unique solution to the bioelectromagnetic inverse problem. We showed that electric fields and potentials within the brain are predominantly due to ohmic currents. On this basis we reformulated the inverse problem in terms of a restricted source model that allows one to noninvasively estimate Local Field Potentials (LFPs) in depth from scalp data. Incorporating as a priori information the physical laws predicting the decay of the strength of potential and fields with the distance to their generation site defines a physically driven regularization strategy that achieves uniqueness in the solution. Consequently, both the regularization strategy and the source model emulate the behavior of the actual generators of brain activity.

The introduction of biophysical constraints into the inverse problem yields to estimate of LFP that are consistent in terms of their spatiotemporal features with experimental evidence available from recordings in humans and animals. The basic functionally active areas detected by this inverse solution are in accordance with the results obtained from experimental physiology and independent functional localization techniques (fMRI). Due to the high temporal resolution and the quality of the temporal estimates, we believe that the noninvasive estimation of LFP based on biophysical constraints will become a useful tool for the study of brain function in healthy subjects. Advances in our understanding of the principles underlying the brain functional networks depend upon understanding the flow of information and the functional interactions between distant brain regions. LFPs are easier to understand, treat and evaluate than current density estimates, which constitutes another appealing reason to use this model for the solution of the inverse problem. Methods similar to the ones used to analyze oscillatory neural phenomena, or binding mechanism in intracranial recordings can be applied to these inverse estimates provided that the temporal estimates are reliable. The results presented in this paper and references therein are a step forward in confirming its reliability.

\section{Acknowledgments}

Swiss National Foundation Grants 3152A0-100745 and the MHV Foundation Grant 3234-069264 supported this work. Part of this research was conducted under support of a IM2 white paper grant.

\section{Appendix A}

This appendix shows that for any media where the Green function exists, the current density vector of a dipole is an irrotational vector field. For that, consider the relationship between the Green function of the Laplacian operator associated to the geometry under consideration and the Dirac delta function. Under this formalism, the current density vector of a dipole located at point $p$ can be written as:

$$
\begin{aligned}
\boldsymbol{J}(r) & =M \cdot \delta(r-p)=-M \cdot \nabla_{r}^{2} G(r, p) \\
& =\nabla_{r}\left(-M \cdot \nabla_{r} \mathrm{G}(r, p)\right)
\end{aligned}
$$

Where $\nabla_{r}$ and $\nabla_{r}^{2}$ represents the gradient and the laplacian with respect to variable $r$. Previous expression shows that the current density vector of a dipole can be written as the gradient of a scalar function and thus by definition it is irrotational.

\section{Appendix B}

This appendix give the details to implement the method described in this paper for the case of the current density vector (3D vector field) and the LFP (scalar field).

(a) Denote by $V_{i}$ the vicinity of each solution point, defined as the hexaedron centered at the point and comprising at most $N=26$ neighbors.

(b) For each solution point $k$, denote by $N_{k}$ the number of neighbors of that point and by $d_{k i}$ the Euclidean distance form point $k$ to point $i$ (or vice versa).

(c) Compute the elements of matrix $\mathbf{A}$ using $e_{i}=2$ for scalar fields and $e_{i}=3$ for vector fields.

$$
\boldsymbol{A}_{i i}=\frac{N}{N_{i}} \sum_{k \subset V_{i}} d_{k i}^{-e_{i}} \quad \boldsymbol{A}_{i k}=-d_{k i}^{-e_{i}}
$$

(d) Define the diagonal matrix $\mathbf{W}$ for the scalar field as the identity matrix and for a vector field as the mean of the norm of the three columns of the lead field matrix associated with point $i$.

(e) Compute matrix $\mathbf{M}=\mathbf{W A}$ (for scalar field) and $\mathbf{M}=\mathbf{W A} \otimes \mathbf{I}_{3}$ (for vector field) where $\otimes$ denotes the Kronecker product of matrices and $\mathbf{I}_{3}$ stands for the identity matrix of dimension 3 .

(f) Define the metric in the source space as $\mathbf{W}_{j}=\left(\mathbf{M}^{\mathrm{t}} \mathbf{M}\right)^{-1}$ and the metric in the data space as the Identity matrix (if available use the inverse of the covariance matrix of the data).

(g) Compute the inverse matrix using Eq. (13) for the noise-free case or Eq. (11) for the noisy data. If no a priori information is available use $\boldsymbol{J}_{\mathrm{p}}=0$.

\section{Appendix C}

The Bonferroni method to correct $P$ values is known to be very conservative. For the case of inverse solutions estimated from $N_{\mathrm{s}}$ independent sensors it is not difficult to see that the maximum number of independent estimates in the source space cannot be higher than $N_{\mathrm{s}}$. On this basis we propose to correct the $P$ values for multiple tests by the number of independent sensors instead of using the number of tests. In our case that corresponds to use $N_{\mathrm{s}}=$ 110 instead of $N_{\mathrm{p}}=4024$, that is, more than one order less.

\section{References}

Arieli, A., Shoham, D., Hildesheim, R., Grinvald, A., 1995. Coherent spatiotemporal patterns of ongoing activity revealed by real time optical imaging coupled with single unit recording in the cat visual cortex. J. Neurophysiol. 73, 2072-2093. 
Bablioni, F., Bablioni, C., Carducci, F., Romani, G.L., Rossini, P.M., Angelone, L.M., Cincotti, F., 2003. Multimodal integration of high-resolution EEG and functional magnetic resonance imaging data: a simulation study. NeuroImage 19, 1-15.

Ben-Israel, A., Greville, T.N.E., 1974. Generalized Inverses: Theory and Applications. Wiley, New York.

Bertero, M., De Mol, C., Pike, E.R., 1985. Linear inverse problems with discrete data: I. General formulation and singular system analysis. Inverse Problems 1, 301-330.

Buchner, H., Gobbelé, R., Wagner, F., Fuchs, M., Waberski, T.D., Beckmann, R., 1997. Fast visual evoked potential input into human area V5. NeuroReport 8, 2419-2422.

Dale, A.M., Halgren, E., Lewine, J.D., Buckner, R.L., Fischl, B., Marinkovic, K., Paulson, K.M., Oostendorp, T.F., Belliveau, J.W., Orrison, W., Rosen, B.R., 2000. Spatiotemporal cortical activation patterns during semantic processing of novel and repeated words as revealed by combined fMRI and MEG. Neuron (Neurotechnique) 26, 55-67.

Dale, A.M., Sereno, M.I., 1993. Improved localization of cortical activity by combining EEG and MEG with MRI cortical surface reconstruction: A linear approach. J. Cogn. Neurosci. 5, 162-176.

Donchin, O., Gribova, A., Steinberg, O., Bergman, H., Cardoso de Oliveira, S., Vaadia, E., 2001. Local field potentials related to bimanual movements in the primary and supplementary motor cortices. Exp. Brain Res. 140, 46-55.

Ducommun, C.Y., Murray, M.M., Thut, G., Bellmann, A., Viaud-Delmon, I., Clarke, S., Michel, C.M., 2002. Segregated processing of auditory motion and auditory location: An ERP mapping study. NeuroImage 16, $76-88$.

Ffytche, D.H., Guy, C.N., Zeki, S., 1995. The parallel visual motion inputs into areas V1 and V5 of human cerebral cortex. Brain 118, 1375-1394.

Fuchs, M., Wagner, M., Köhler, T., Wischmann, H.A., 1999. Linear and nonlinear current density reconstructions. J. Clin. Neurophysiol. 16, 267-295.

Grave de Peralta Menendez, R., Gonzalez Andino, S.L., 1998. A critical analysis of linear inverse solutions. IEEE Trans. Biomed. Eng. 4, $440-448$.

Grave de Peralta Menendez, R., Gonzalez Andino, S.L., 1999. Distributed source models: standard solutions and new developments. In: Uhl, C. (Ed.), Analysis of Neurophysiological Brain Functioning. Springer Verlag, Berlin Heidelberg, pp. 176-201.

Grave de Peralta Menenedez, R., Gonzalez Andino, S.L., 2002. Comparison of algorithms for the localization of focal sources: evaluation with simulated data and analysis of experimental data. Int. J. Bioelectromagn., 4.

Grave de Peralta Menendez, R., Gonzalez Andino, S.L., Morand, S., Michel, C.M., Landis, T.M., 2000. Imaging the electrical activity of the brain: ELECTRA. Hum. Brain Mapp. 1, 1-12.

Grave de Peralta Menendez, R., Gonzalez Andino, S.L., Lantz, G., Michel, C.M., Landis, T., 2001. Noninvasive localization of electromagnetic epileptic activity. I. Method descriptions and simulations. Brain Topogr. 14, 131-137.

Greenblatt, R.E., 1993. Probabilistic reconstruction of multiple sources in the neuroelectromagnetic inverse problem. Inverse Problems 9, $271-284$.

Groetch, C.W., 1984. The Theory of Tikhonov Regularization for Fredholm Equations of the First Kind. Pitman, Boston.

Hämäläinen, M.S., Ilmoniemi, R.J., 1984. Interpreting measured magnetic fields of the brain: Estimates of current distributions. Technical Report TKK-F-A559, Helsinki University of Technology.

Hämäläinen, M.S., Hari, R., Ilmoniemi, R.J., Knuutila, J., Lounasma, O.V., 1993. Magnetoencephalography-theory, instrumentation, and applications to non invasive studies of the working human brain. Rev. Mod. Phys. 65, 413-497.

Hauk, O., Keil, A., Elbert, T., Müller, M.M., 2002. Comparison of data transformation procedures to enhance topographical accuracy in time-series analysis of the human EEG. J. Neurosci. Methods 113, $111-122$
Khateb, A., Michel, C.M., Pegna, A., O’Dochartaigh, S.D., Landis, T., Annoni, J.M., 2003. Processing of semantic categorical and associative relations: an ERP mapping study. Int. J. Psychophysiol. 49, $41-55$.

Liu, A.K., Dale, A.M., Belliveau, J.W., 2002. Monte Carlo simulation studies of EEG and MEG localization accuracy. Hum. Brain Mapp. $16,47-62$.

Logothetis, N.K., Pauls, J., Augath, M., Trinath, T., Oeltermann, A., 2001. Neurophysiological investigation of the basis of the fMRI signal. Nature 412, 150-157.

Malmivuo, J., Plonsey, R., 1995. Bioelectromagnetism-Principles and Applications of Bioelectric and Biomagnetic Fields. Oxford Univ. Press, New York.

Menke, W., 1989. Geophysical Data Analysis: Discrete Inverse Theory. Academic Press, San Diego, CA.

Michel, C.M., Seeck, M., Murray, M.M., 2003. The speed of visual cognition. In: Hallett, M., Phillips, L., Schomer, D., Massey, J. M. (Eds.), Supplement series to Clinical Neurophysiology, 2003 (in press).

Michel, C.M., Grave de Peralta, R., Lantz, G., Gonzalez Andino, S.L., Spinelli, L., Blanke, O., Landis, T., Seeck, M., 1999. Spatio-temporal EEG analysis and distributed source estimation in presurgical epilepsy evaluation. J. Clin. Neurophysiol. 16, 239-266.

Michel, C.M., Thut, G., Morand, S., Khateb, A., Pegna, A.J., Grave de Peralta, R., Gonzalez, S., Seeck, M., Landis, T., 2001. Electric source imaging of human cognitive brain functions. A review. Brain Res. Rev. 36, $108-118$.

Mitzdorf, U., Singer, W., 1977. Laminar segregation of afferents to lateral geniculate nucleus of the cat: an analysis of current source density. J. Neurophysiol. 40, 1227-1244.

Morand, S., Thut, G., Grave de Peralta, R., Clarke, S., Khateb, A., Landis, T., Michel, C.M., 2000. Electrophysiological evidence for fast visual processing through the human koniocellular pathway when stimuli move. Cereb. Cortex 10, 817-825.

Mosher, J., Mosher, R., Leahy, F., Lewis, P., 1992. Multiple dipole modeling and localization from spatiotemporal MEG data. IEEE Trans. Biomed. Eng., 541-557 (June).

Murray, M.M., Foxe, J.J., Higgins, B.A., Javitt, D.C., Schroeder, C.E., 2001. Visuo-spatial neural response interactions in early cortical processing during a simple reaction time task: a high-density electrical mapping study. Neuropsychologia 39, 828-844.

Murray, M.M., Wylie, G.R., Higgins, B.A., Javitt, D.C., Scroeder, C.E., Foxe, J.J., 2002. The spatio-temporal dynamics of illusory contour processing: Combined high-density electrical mapping, source analysis, and functional magnetic resonance imaging. J. Neurosci. 22, $5055-5073$.

Murray, M.M., Michel, C.M., Grave de Peralta, R., Ortigue, S., Brunet, D., Gonzalez Andino, S., Schnider, A., 2003. Rapid discrimination of visual and multisensory memories revealed by electrical neuroimaging. NeuroImage (in press).

Parker, R.L., 1994. Geophysical Inverse Theory. Princeton Univ. Press, Princeton, NJ.

Pegna, A.J., Khateb, A., Murray, M.M., Landis, T., Michel, C.M., 2002. Neural processing of illusory and real contours revealed by high density ERP mapping. NeuroReport 13, 965-968.

Penrose, R., 1955. A generalized inverse for matrices. Proc. Cambridge Philos. Soc. 51, 406-413.

Phillips, C., Rugg, M.D., Friston, K.J., 2002. Anatomically informed basis functions for EEG source localization: combining functional and anatomical constraints. NeuroImage 16, 678-695.

Plonsey, R., 1982. The nature of the sources of bioelectric and biomagnetic fields. Biophys. J. 39, 309-312.

Rao, C.R., Mitra, S.K., 1971. Generalized inverse of matrices and its applications. Wiley, New York.

Roach, G.F., 1970. Green Functions. Introductory Theory with Applications. Van Nostrand Reinhold, London.

Rorden, C., Brett, M., 2000. Stereotaxic display of brain lesions. Behav. Neurol. 12, 191-200. 
Sarvas, J., 1987. Basic mathematical and electromagnetic concepts of the bioelectromagnetic inverse problem. Phys. Med. Biol. 32, 11-22.

Scherg, M., 1990. Fundamentals of dipole source potential analysis. In: Grandori, F., Hoke, M., Romani, G. (Eds.), Auditory Evoked Magnetic Fields and Electric Potentials. Karger, Basel, pp. 40-69.

Schroeder, C.E., Mehta, A.D., Givre, S.J., 1998. A spatio-temporal profile of visual system activation revealed by current source density analysis in the awakw macaque. Cereb. Cortex 8, 575-592.

Schwartz, D.P., Liu, A.K., Bonmassar, G., Belliveau, J., 2001. Comparison of the Linear Estimation and Spatio-temporal Fit Inverse Approaches. Int. J. Bioelectromagn. 3.

Sekihara, K., Nagarajan, S.S., Poeppel, D., Marantz, A., Miyashita, Y., 1999. Application of an MEG eigenspace beamformer to reconstructing spatio-temporal activities of neural sources. Hum. Brain Mapp. 15, $199-215$.

Stok, C., 1987. The influence of model parameters on EEG/MEG single dipole source estimation. IEEE Trans. Biomed. Eng. 34, 289-296.

Thut, G., Hauert, C.A., Blanke, O., Morand, S., Seeck, M., Gonzalez, S.L., Grave de Peralta, R., Spinelli, L., Khateb, A., Landis, T., Michel, C.M., 2000. Visually induced activity in human frontal motor areas during simple visuomotor performance. NeuroReport 11, 2843-2848.

Tikhonov, A.M., Arsenin, V.Y., 1977. Solutions of Ill-Posed Problems. Wiley, New York.

Uutela, K., Hamalainen, M., Somersalo, E., 1999. Visualization of magnetoencephalographic data using minimum current estimates. NeuroImage $10,173-180$ 